Article

\title{
Investigation of Copper, Zinc and Strontium Doping on Elec- trochemical Properties of Titanium Dioxide Nanotubes for In Vitro Neural Recording
}

\author{
Dhurgham Khudhair ${ }^{a 1}$, Julie Gaburro ${ }^{b 2}$, Hoda Amani Hamedani ${ }^{c 3}$, Anders Barlow ${ }^{d 4}$, Hamid Garme- \\ stani ${ }^{e 5}$ and Asim Bhatti ${ }^{a 1 *}$

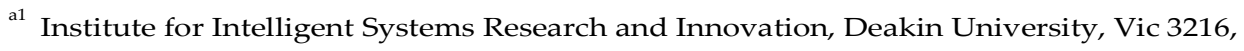 \\ Australia \\ b2 Australian Animal Health Laboratory - CSIRO VIC 3219, Australia \\ ${ }^{c 3}$ Department of Materials Science and Engineering, Case Western Reserve University, \\ Cleveland, $\mathrm{OH} 44106$, USA \\ ${ }^{\mathrm{d} 4}$ Centre for Materials and Surface Science, La Trobe University, Bundoora, VIC, 3086, Aus- \\ tralia \\ ${ }^{\text {e5 }}$ School of Material Sciences and Engineering, Georgia Institute of Technology, GA 30332, \\ USA \\ * Correspondence: asim.bhatti@deakin.edu.au
}

\begin{abstract}
Direct interaction with the neuronal cells is a prerequisite to deciphering useful information in understanding the underlying causes of diseases and functional abnormalities in the brain. Precisely fabricated nanoelectrodes provide the capability to interact with the brain in its natural habitat without compromising its functional integrity. Considerable research has been focused on the employment of vertical nanotubes as nanoelectrodes due to large-scale intracellular recording capability and longer-term intracellular access that arise from their unique geometry. Despite many types of nanotube structures reported in the literature, a limited subset of the nanotubes has been investigated for neural interfacing. This work reports on the fabrication and optimisation of vertically oriented titania nanotube arrays as a scalable electrode platform for neural interface application. To this end, the doping was performed by incorporating a selected group of biologically active metallic ions, including zinc, strontium, and copper, into $\mathrm{TiO} 2$ lattice and its effect was studied with respect to the structural, electrochemical and biological properties of the nanotube arrays. It was found that doping can change the length, diameter and wall thickness of the nanotubes. Among pure and doped samples, the copper-doped $\mathrm{TiO} 2$ nanotubes demonstrated the highest electrochemical and biological performance. Our results suggest that the doping can be used as a promising method to optimise the properties of nanotube arrays for the development of high-performance neural electrodes.
\end{abstract}

Keywords: Neural Interface; $\mathrm{TiO}_{2}$ Nanotube arrays; Biocompatibility; Electrochemical Properties; Doping

\section{Introduction}

Understanding the dynamics and complexity of the brain functions at the neural cell level is one of the main challenges of neuroscience. To this end, previous works have demonstrated a promising approach for precise recordings of neural activities over a wide range of brain circuits based on implanting multi-electrode array devices [1]. For neural stimulation, electrodes should be capable of injecting relatively large currents with minimum electrode degradation resulting from of faradic effects. This can be achieved by increasing the electrode size, which is often accompanied by a negative impact on the biocompatibility. For long-term stimulation, the 
large surface area of the electrode is required to facilitate charge transport and reduce inflammatory reaction and gliosis [2]. The nanoscale dimensions of neuron elements such as the cytoskeleton, ionic channels, and filopodia make materials with nanostructures the most suitable architectures to accommodate these features [3, 4]. Recently, nanoscale electrodes of various vertical geometries have been shown as promising neural interfaces. To this end, nanowires of platinum, gold and gallium phosphide have been used to record electrical activities from neural systems [5, 6, 7]. Heo et al. have shown stable neural signal recording using polyimide nanofibers [8]. In vitro, vertically aligned carbon nanofibers were developed as a neural chip to stimulate and monitor electrical signals from brain tissue [9].

In this regard, nanomaterials-based approaches for improving electrode functions as well as their biological response have received considerable attention. Among various morphologies of vertical nanoelectrodes, vertically oriented nanotube arrays have shown great potential for the next generation of electrodes for neural interface applications. This surpassing progress is made possible by the unique geometry of the vertically oriented nanotube arrays as well as their one-dimensional pore structure with high surface area, which promotes cell-electrode electrochemical coupling and provides prolonged access to the cell interior [10]. Previous observations have shown that the cell membranes have a tendency to wrap around the nanoobjects and protrude outward into nanoholes as small as $100 \mathrm{~nm}$ diameter [11].

Among different materials of similar morphology, nanotubes of a narrow range of materials limited to iridium oxide and carbon have been employed for the application of neural interfacing $[10,12,2]$. High electrochemical impedance, toxicity and fabrication complexity are the main limitations of metal oxide nanotubes hindering their use in neural interface application [13]. Vertically oriented $\mathrm{TiO}_{2}$ nanotube arrays have been widely studied as a promising biocompatible material for a broad range of biomedical applications $[14,15,16]$. Many previous studies have suggested $\mathrm{TiO}_{2}$ nanotubes as a favorable material for orthopedic and dental implants as well as drug delivery membranes $[14,17]$. Among all, the high impedance and low charge storage capacitance of $\mathrm{TiO}_{2}$ nanotubes limit their use as electrodes in neural interfacing. Tube morphology was pointed out as a crucial factor affecting the electrochemical properties and biocompatibility of $\mathrm{TiO}_{2}$ nanotubes [13]. Doping with metallic and non-metallic elements has been employed to improve the biocompatibility of $\mathrm{TiO}_{2}$ nanotube arrays $[18,16]$. For some non-medical applications such as photocatalysis, dye sensitized and solar cells, annealing and doping with various types of elements have been employed to enhance the electrochemical properties of nanotubearrays $[19,20]$. Besides titania, zinc oxide $(\mathrm{ZnO})$ nanostructures have attracted much attention due to their importance in health care applications and their wide range of biological activities, as well as the low cost of their fabrication $[21,22]$. Previous studies have also shown that doping with strontium significantly improves the biological and electrochemical properties of $\mathrm{TiO}_{2}$ nanotubes [23, $24]$. On the other hand, copper doping has shown to improve cell regeneration in glass ceramics and increase the hydrophilicity of $\mathrm{TiO}_{2}$ nanotube arrays $[25,26]$. However, tailoring the structure of $\mathrm{TiO}_{2}$ to achieve a combination of low electrochemical impedance, high charge storage capacitance and good biocompatibility remains challenging. In this work, we report on the fabrication and optimization of vertically oriented nanotube arrays of doped titania as a potential electrode platform for parallel electrical interfacing to neurons. First, the effect of zinc, Strontium, and copper doping on the structure and electrochemical properties of $\mathrm{TiO}_{2}$ nanotube arrays are studied. Then, the wettability and biocompatibility of the doped $\mathrm{TiO}_{2}$ nanotube arrays were evaluated using contact angle measurements and cell culture tests.

\section{Materials and Methods}

\subsection{Fabrication of the Nanotube Arrays}


Electrochemical anodization was used to prepare $\mathrm{TiO}_{2}$ nanotube arrays were. Pure titanium foils ( $1.5 \mathrm{~cm} \times 1 \mathrm{~cm} \times 50 \mu \mathrm{m}$, Sigma Aldrich purity $\geq 99.7 \%)$ were ultrasonically cleaned with acetone, ethanol and deionized (D.I.) water for 10 minutes. The cleaned foils were subjected to a constant voltage of $40 \mathrm{~V}$ in an electrochemical anodization cell. Foils of titanium served as anode and standard platinum electrode as the cathode. The cathode and the anode were separated with a distance of $2 \mathrm{~cm}$. The electrolyte used for the anodization of pure $\mathrm{TiO}_{2}$ nanotubes was prepared by dissolving $0.5 \mathrm{~g}$ of $\mathrm{NH}_{4} \mathrm{~F}$ in $4 \mathrm{ml}$ of D.I. water, then the resultant solution was mixed with $94 \mathrm{ml}$ of ethylene glycol (EG, anhydrous, Sigma Aldrich 99.8\%).

In-situ doping was performed during the electrochemical anodization process. Using the same electrolyte, the zinc, strontium and copper-doped titania (i.e., $\mathrm{Zn}$ doped, Sr-doped and $\mathrm{Cu}$-doped $\mathrm{TiO}_{2}$ ) nanotube arrays were fabricated by adding 0.01 $\mathrm{M} \mathrm{Zn}\left(\mathrm{NO}_{3}\right)_{2}, 0.06 \mathrm{M} \mathrm{Sr}(\mathrm{OH})_{2}$ and different concentrations of $\mathrm{Cu}\left(\mathrm{NO}_{3}\right)_{2}$ into the electrolyte. For copper-doped nanotubes, the electrolyte consisting of three different concentrations of $\mathrm{Cu}\left(\mathrm{NO}_{3}\right)_{2}(0.01 \mathrm{M}, 0.02 \mathrm{M}$ and $0.03 \mathrm{M})$ was prepared to evaluate the effect of copper ions concentrations. Doped nanotube arrays were anodized under the same conditions used for the preparation of pure nanotube arrays. Pure and doped nanotube samples were annealed at $500{ }^{\circ} \mathrm{C}$ at heating rate of $4{ }^{\circ} \mathrm{C}$ /min for 3 hours in an atmosphere of nitrogen using a tube furnace (Tetlow furnaces, with a FP93 Controller/Programmer).

\subsection{Characterization of the Annealed Nanotube Arrays}

The morphology of fabricated $\mathrm{TiO}_{2}$ nanotubes was characterized using Ultra High-Resolution Field Emission Scanning Electron Microscopy (FE-SEM, Carl Zeiss SUPRA ${ }^{\circledR}$ ). Inner diameters of ten nanotubes were measured for each sample using ImageJ software and the mean value was calculated. The crystal structure was investigated by X-ray Diffraction (XRD, Panalytical X-Pert Pro MRD XL, $\mathrm{CuK} \alpha$ radiation, $\lambda=1.5418 \AA$ ) Surface elemental analysis was carried out using X-ray Photoelectron Spectroscopy (XPS) by the Kratos AXIS Nova instrument (Kratos Analytical Ltd, U.K.) equipped with a monochromated $\mathrm{Al} \mathrm{K} \alpha$ radiation source (1486.69 eV) operating at $150 \mathrm{~W}$ power $(15 \mathrm{kV}, 10 \mathrm{~mA})$, on area of $\sim 300 \mu \mathrm{m} \times 700 \mu \mathrm{m}$. To identify the elemental composition, survey spectra were obtained at $160 \mathrm{eV}$ pass energy. Pass energy of $20 \mathrm{eV}$ was applied to collect high-resolution spectra to identify the oxidation states.

\subsection{Electrochemical Properties of the Fabricated Nanotube Arrays}

Electrochemical impedance spectroscopy (EIS) and cyclic voltammetry (CV) tests were performed on $\mathrm{TiO}_{2}$ nanotubes in a three-electrode setup using a potentiostat (Bio-Logic Science Instruments, Model VSP). The nanotube film on the titanium substrate as a working electrode, platinum standard electrode as a counter electrode and saturated $\mathrm{Ag} / \mathrm{AgCl}$ electrode as a reference electrode were used in a potassium buffered saline solution $(3 \mathrm{M}, \mathrm{KCl})$. For data acquisition and analysis, the software (Bio-Logic EC-lab) was used. Bode plots were utilized to identify electrochemical impedance. $\mathrm{CV}$ of the nanotubes was performed under an applied voltage range of -100 $\mathrm{mV}$ to $600 \mathrm{mV}$ versus an $\mathrm{Ag} / \mathrm{AgCl}$ reference electrode. $\mathrm{CV}$ of the fabricated samples were conducted at a scanning rate of $100 \mathrm{mV} / \mathrm{s}$ in electrolyte of $3 \mathrm{M} \mathrm{KCl}$. The sample area under test was $4.9 \mathrm{~mm}^{2}$ in all electrochemical experiments, which was obtained by calculating the circular area of the exposed region. The electrochemical measurements were repeated three times for each sample.

\subsection{Contact Angle Measurements}

Static contact angle measurements were carried out on the fabricated nanotube films by an optical contact angle meter (Cam 200, KSV Instruments Ltd., Finland) using water droplet with a volume of $(\sim 3 \mathrm{ml})$ on every sample at room temperature and in an ambient 
atmosphere to evaluate the wettability of the nanotube films. Photos were captured to record the images of the water drops. The contact angles of the water drop on the nanotube samples were measured in the recorded images using the KSV-Cam software (5 repeats for each sample).

\subsection{Vero Cell Culture on the Fabricated Nanotube Arrays}

Cytotoxicity studies were performed using Vero cells. The 'Vero' lineage was isolated from kidney epithelial cells extracted from an African green monkey (ATCC CCL-81). The use of Vero cells to characterize the biocompatibility of materials is well-established practice in material science. This is due to the fact that Vero cells are considered to be one of the most suitable cells for cytotoxicity studies and for the analysis of cell-substrate interactions in biomaterial research [26, 27]. For sterilization, nanotube samples were individualized in a $2 \mathrm{ml}$ glass tube and heated in an autoclave for 45 minutes at $121^{\circ} \mathrm{C}$. and were dried by an air gun. The coating process included implementing $200 \mu \mathrm{l}$ of polyethyleneimine for 30 minutes. Then, samples were rinsed three times, with tissue culture grade water. The rinsed samples were treated with laminin by spreading $50 \mu$ l of laminin on each sample surface. Then, samples were placed in an incubator with $5 \% \mathrm{CO}_{2}$ for 30 minutes at a $37^{\circ} \mathrm{C}$. After removing the residue laminin, Vero cells were seeded by adding $1 \mathrm{ml}$ of media solution to the surface of each sample and let to rest in the incubator for 2-, 4-, and 7-days. The experiment was repeated three times for each sample and the standard deviation from each mean value was calculated.

\subsection{Mouse Embryo Dissection procedure}

Cortical embryo primary neurons from mouse, Mus musculus were prepared by the tissue culture laboratory at the Australian Animal Health Laboratory (AAHL-CSIRO) under the permit AEC number 1686. Whole brains were extracted from C57BL/B6 mouse embryos at embryonic day 15 (E15). Cortical neurons from embryos were removed aseptically from the brain by gently removing the meninges in cold dissection medium Hibernate $\left(\mathrm{Gibco}^{\circledR}\right)$. To perform enzymatic dissociation, isolated cortex hemispheres were then treated at $37^{\circ} \mathrm{C}$ with Deoxyribonuclease (DNase) I Solution $(0.75 \mathrm{mg} / \mathrm{ml})$ and Trypsin in Minimal Essential Medium (MEM) (5 mg/ml) for 5-10 min. Then, mechanical dissociation was performed after three washes with the dissection medium by 10 passages through a $10 \mathrm{ml}$ glass pipette. The cell suspension was then centrifuged for $5 \mathrm{~min}$ at $100 \times \mathrm{g}$, and the pellet was re-suspended in supplemented Neurobasal ${ }^{\circledR}$ culture medium (ThermoFisher ${ }^{\circledR}$ ). The experiment was repeated three times for each sample and the standard deviation from each mean value was calculated.

\subsection{Cortical Neurons Culture on the Fabricated Nanotube Arrays}

Following the same protocol as for the Vero cell culture, cortical neurons were seeded. Samples were sterilized, dried, coated with polyethylenimine (PEI) and rinsed with TC water. Then, laminin was spread over the sample surface. After removing of the remaining of laminin, a volume of dissociated neurons from the stock solution is gently added to the samples, without any solution going over the edges. The plate is placed in an incubator with $5 \% \mathrm{CO}_{2}$ for 1 hour at $37{ }^{\circ} \mathrm{C}$ to let the cells adhere to the samples. Each well is then completed with $1 \mathrm{ml}$ of Neurobasal ${ }^{\circledR}$ culture media. An indirect cell count of the neurons growing on the nanotube samples is made at 3-days post-seeding.

\subsection{Cell Counting}

Due to the lack of transparency of the nanotube films to observe the cells under the microscope, an indirect cell counting procedure is performed at 2-, 4-, and 7-days post Vero cells seeding, and 3-days post primary neurons seeding on the samples. For that, samples were carefully removed from the well with forceps and rinsed with Phosphate Buffer Saline (PBS). A volume of $100 \mu$ of Trypsin/EDTA diluted at 1:10 in PBS was added on the samples in a new 24-well plate. The samples were let to rest in an incubator for 5 
minutes for enzyme activation. To stop enzyme activity, either $500 \mu \mathrm{l}$ of Dulbecco's Modified Eagle's Medium (DMEM) was added to the Vero cell culture, or $100 \mu$ l of Neurobasal ${ }^{\circledR}$ media to the primary neuron culture. Volumes in wells were rigorously mixed to detach the cells from the samples.

For cell counting a volume of $30 \mu \mathrm{l}$ of the cell solution was taken and counted under the microscope with a hemocytometer for each sample. Using a hand tally counter, cells were counted and the total number of neurons on the samples was estimated by the following formula:

Number of cells per $m l=\frac{\text { number of counted cells } \times \text { dilution factor } \times 10,000}{\text { number of squares counted on the hemocytometer }}$

\section{Results and Discussion}

\subsection{Structure and Morphology of the Fabricated Nanotube Arrays}

Figure 1 shows the top view and cross-sectional SEM images (shown in the insets) of pure (Figure $1 \mathrm{a}$ ) and doped (Figure $2 \mathrm{~b}-\mathrm{f}$ ) $\mathrm{TiO}_{2}$ nanotube arrays after annealing. It is evident that the fabricated nanotubes are vertically ordered. The nanotubes dimensions were measured using imageJ software. For pure nanotubes (as-fabricated after annealing), the average tube diameter, tube length and tube wall thickness are $61 \mathrm{~nm}, 2.25 \mu \mathrm{m}$ and $25 \mathrm{~nm}$, respectively (Figure 1-a). Figure $1 \mathrm{~b}$ shows the top view and cross-sectional SEM images of $\mathrm{Zn}$-doped nanotube arrays. The average tube diameter, tube length and the tube wall thickness of Zn-doped nanotube arrays are $67 \mathrm{~nm}, 1.1 \mu$ and $15 \mathrm{~nm}$, respectively. The measurements revealed that zinc doping leads to a slight increase in the tube diameters compared to those of pure nanotubes fabricated at the same voltage, while a significant decrease in the tube length and tube wall thickness was observed.

Top and cross-sectional SEM images of Sr-doped nanotubes are shown in Figure 1c. The average tube diameter, tube length and the tube wall thickness of Sr-doped nanotubes were $67.4 \mathrm{~nm}, 1.8 \mu \mathrm{m}$ and $23 \mathrm{~nm}$, respectively. It was observed that larger diameter, shorter length and thinner wall resulted from doping of nanotubes with strontium as compared to pure nanotubes. Characterization of the tube morphology of Cu-doped nanotubes revealed that both the tube diameter and the tube length decreased while tube wall thickness increased after adding copper nitrate to the electrolyte. Figure 1d shows the SEM images of $\mathrm{Cu}$-doped nanotube arrays fabricated in electrolyte containing $0.01 \mathrm{M}$ copper nitrate $\left(\mathrm{Cu}\right.$-doped $\left.\mathrm{TiO}_{2}(0.01 \mathrm{M})\right)$. The measurements were found to be $60 \mathrm{~nm}$ for tube diameter, $1.3 \mu \mathrm{m}$ for tube length and $32.2 \mathrm{~nm}$ for tube wall thickness. By increasing the concentration of copper nitrate to $0.02 \mathrm{M}$ in the electrolyte, the average inner tube diameter, tube length and tube wall thickness changed to approximately $56 \mathrm{~nm}, 1 \mu \mathrm{m}$ and 38 $\mathrm{nm}$, respectively. The top and cross-sectional SEM images of the nanotube arrays fabricated in the electrolyte containing $0.02 \mathrm{M}$ copper nitrate $\left(\mathrm{Cu}\right.$-doped $\mathrm{TiO}_{2}(0.02 \mathrm{M})$ ) are shown in Figure 1e. It was found that increasing the concentration of the copper nitrate to $0.03 \mathrm{M}$ leads to the blockage of the nanotube pores (Figure 1f). As the opened pores of the nanotubes are the key aspect of their biological application, $\mathrm{Cu}$-doped nanotubes with $0.03 \mathrm{M}$ of dopant concentration were excluded from further investigation.

To investigate the structure of the doped nanotube arrays after annealing, samples were subjected to XRD analysis. Figure 2 shows the XRD patterns of the fabricated nanotube arrays. The XRD pattern of annealed nanotubes shows a crystal structure with anatase phase recognized by the peaks (and the corresponding crystallographic plane) at $2 \theta$ $=$ of $25.418^{\circ}(101), 38.765^{\circ}(112), 48.22^{\circ}(200), 54.344^{\circ}$ (105) and 55.292 ${ }^{\circ}$ (211). The peak positions are consistent with the standard powder diffraction data for anatase (JCPDS card \#01-070-6826), Titanium (JCPDS card \#01-071-4632) and rutile (JCPDS) card \#00-021-1276). The patterns of the doped nanotube arrays show a clear shift to the lower angles in anatase peaks compared to the pattern of the annealed pure nanotube arrays. This shift is due to the incorporation of dopant ions with an ionic radius of $0.88 \AA, 1.32 \AA$, and $0.87 \AA$, for $\mathrm{Zn}^{2+}, \mathrm{Sr}^{2+}$ and $\mathrm{Cu}^{2+}$, respectively, which are larger than that of $\mathrm{Ti}^{4+}$ 
$(0.75 \AA)$ into the lattice of the titanium oxide causing strain and disorder in the crystal lattice structure.

For chemical state inspection, the XPS analysis was performed on the pure and doped nanotube arrays. High-resolution spectra of Ti $2 p, \mathrm{Zn} 2 \mathrm{p}, \mathrm{Sr} 3 \mathrm{~d}$ and $\mathrm{Cu} 2 \mathrm{p}$ are displayed in Figure 3. It can be seen that the $\mathrm{Ti} 2 \mathrm{p}_{3 / 2}$ and $\mathrm{Ti} 2 \mathrm{p}_{1 / 2}$ peaks of pure nanotubes appeared at $458.9 \mathrm{eV}$ and $464.6 \mathrm{eV}$ with a spin orbit splitting of $5.7 \mathrm{eV}$, confirming that both signals correspond to $\mathrm{Ti}^{4+}$ [28] (Figure 3a). It was found that doping with $\mathrm{Zn}$ ions leads to shift in Ti 2 p peaks to higher binding energies, while these peaks shifted to lower energies by doping the nanotube arrays with $\mathrm{Sr}$ and $\mathrm{Cu}$ ions. For the $\mathrm{Zn}$-doped sample, $\mathrm{Zn} 2 \mathrm{p}$ spectra with two peaks obtained corresponding to the $\mathrm{Zn} 2 \mathrm{p}_{3 / 2}$ and $\mathrm{Zn} 2 \mathrm{p}_{1 / 2}$ photoemission spectra occurring at the binding energies of $1023 \mathrm{eV}$ and $1046 \mathrm{eV}$, respectively. As shown in Figure 3b. The two peaks were separated by $23 \mathrm{eV}$, which indicates that $\mathrm{Zn}$ ions in the form of $\mathrm{ZnO}$ clusters are distributed on the surface of $\mathrm{TiO}_{2}$ nanotubes. Similar to previous works, ZnO clusters couldn't be seen on the SEM images. This could be related to the combination of $\mathrm{Ti}-\mathrm{O}-\mathrm{Zn}$ bonds in the lattice of $\mathrm{TiO}_{2}$ as a result of annealing [20, 21]. Figure 3c shows a peak corresponding to the Sr 3d for Sr-doped sample appearing at the binding energy of $133.1 \mathrm{eV}$ indicating that strontium has been successfully doped into $\mathrm{TiO}_{2}$ nanotubes. The Sr 3d binding energy corresponded to the energy level diagram of $\mathrm{Sr}^{2+}$ [23]. Figure $3 \mathrm{~d}$ shows the $\mathrm{Cu} 2 \mathrm{p}$ spectra at $932.3 \mathrm{eV}$, which can be related to the existence of $\mathrm{Cu}^{2+}$ in the $\mathrm{Cu}_{2} \mathrm{O}[29]$.

\subsection{Electrochemical Performance of the Fabricated Nanotube Arrays}

To investigate the electrochemical properties, pure and doped nanotube arrays were subjected to the Electrochemical Impedance Spectroscopy (EIS) and cyclic voltammetry (CV) tests after annealing. Figure 4 a shows Bode plots of fabricated nanotube arrays. The measurement revealed that pure nanotube arrays have an impedance value of $0.501 \mathrm{k} \Omega$. Doping with zinc increased the impedance of resultant nanotube arrays to $2.7 \mathrm{k} \Omega$. The increase in the nanotube impedance could be related to two possible reasons; the first one can be attributed to the tube wall thickness. $\mathrm{Zn}$-doped $\mathrm{TiO}_{2}$ nanotubes have thinner walls than those of pure nanotubes. Previous studies showed that tubes with thicker walls are more conductive than those of thinner ones [13]. The second possible reason can be related to the presence of zinc oxide which have high electrical resistance resulting in decreasing the electrical conductivity of the nanotube arrays.

The EIS results of Sr-doped nanotube arrays showed lower impedance than that those of $\mathrm{Zn}$-doped $\mathrm{TiO}_{2}$ arrays $(1.7 \mathrm{~K} \Omega$ ), but they are still less conductive than the pure nanotubes. Thicker walls of pure nanotubes improve charge transport and reduce the impedance. Furthermore, $\mathrm{Sr}^{2+}$ ions can substitute for $\mathrm{Ti}^{4+}$ in the lattice of $\mathrm{TiO}_{2}$ and in addition to $\mathrm{Ti}^{4+}$ sites, the large-size strontium ions occupy oxygen vacancies adjacent to the substitutional sites [30]. Thus, the number of oxygen vacancies will decrease resulting in a significant increase in the impedance of nanotube arrays.

From Figure 4a, it can be seen that electrochemical impedance of the $\mathrm{TiO}_{2}$ nanotube arrays is significantly decreased after $\mathrm{Cu}$-doping. Impedance values decreased from 0.501 $\mathrm{K} \Omega$ for pure $\mathrm{TiO}_{2}$ nanotubes to $0.331 \mathrm{~K} \Omega$ and $0.295 \mathrm{~K} \Omega$ for $0.01 \mathrm{M}$ and $0.02 \mathrm{M} \mathrm{Cu}$-doping, respectively. The decrease in Impedance values could be attributed to morphological and structural effects. $\mathrm{Cu}$-doped nanotubes have much shorter lengths and thicker walls than those of pure nanotubes. The observed trend seems logical considering that the electrical resistance has direct relationship with the tube length and inverse relationship with the cross-section area [31,32]. Moreover, random substitution of $\mathrm{Cu}^{2+}$ ions in the lattice of $\mathrm{TiO}_{2}$ can contribute to conductivity enhancement. $\mathrm{As}^{\mathrm{Cu}^{2+}}$ is a highly conductive ion, the presence of such ions in the tube walls provides a path with less impedance for the charge transfer compared to the tube walls of copper free $\mathrm{TiO}_{2}$ nanotube arrays. It was observed that increasing the concentration of copper nitrate in the electrolyte from $0.01 \mathrm{M}$ to $0.02 \mathrm{M}$ leads to a slight increase in the conductivity of the resultant nanotubes, possibly due to 
the slight increase in the tube wall thickness, slight decrease in the tube length and more incorporation of $\mathrm{Cu}$ ions in the structure of $\mathrm{TiO}_{2}$.

To estimate charge storage capacitance, cyclic voltammetry measurements were performed on the fabricated nanotube arrays. Figure $4 \mathrm{~b}$ is shows the cyclic voltammetry curves of pure, $\mathrm{Zn}$-doped, Sr-doped and $\mathrm{Cu}$-doped $\mathrm{TiO}_{2}$ nanotube arrays. It was found that charge storage capacitance is inversely proportional to the impedance. Higher capacitance is for the more conductive nanotubes (less electrochemical impedance) and this could be related to low resistance to the charge transfer through the charge-discharge process. It is clear from the areas between charge-discharge curves that $0.02 \mathrm{M} \mathrm{Cu}$-doped $\mathrm{TiO}_{2}$ nanotube arrays have the highest storage capacitance of $130 \mathrm{~F} / \mathrm{g}$. Inversely proportional to the impedance of the nanotube arrays, storage capacitance values were $125 \mathrm{~F} / \mathrm{g}, 94.85$ F/g, $45 \mathrm{~F} / \mathrm{g}$ and $40 \mathrm{~F} / \mathrm{g}$ for $0.01 \mathrm{M} \mathrm{Cu}$-doped $\mathrm{TiO}_{2}$, pure $\mathrm{TiO}_{2}$, Sr-doped $\mathrm{TiO}_{2}$ and $\mathrm{Zn}$-doped $\mathrm{TiO}_{2}$, respectively.

\subsection{Wettability of the Fabricated Nanotube Arrays}

To estimate the wettability of the doped nanotube arrays, water contact angle was measured for pure, $\mathrm{Zn}$-doped, $\mathrm{Sr}$-doped and $\mathrm{Cu}$-doped $\mathrm{TiO}_{2}$ nanotube arrays. The chart in Figure 5 shows the static water contact angle of the water droplets on the surfaces of the pure and doped $\mathrm{TiO}_{2}$ nanotube arrays with their corresponding images of the water droplets on the samples shown on top. Except for $\mathrm{Zn}$-doped $\mathrm{TiO}_{2}$ nanotubes $\left(\theta>90^{\circ}\right)$, all samples showed a hydrophilic property $\left(\theta<90^{\circ}\right)$. Cu-doped nanotube arrays $(0.02 \mathrm{M})$ exhibited the highest hydrophilicity. This can be due to more oxygen vacancies introduced induced in the lattice of $\mathrm{TiO}_{2}$ by doping with $\mathrm{Cu}^{2+}$ ions. These vacancies are favourable sites for occupation by water molecules; this enables the rapid spread of water on the surface [24]. Sr-doped nanotube arrays showed lower hydrophilicity than Cu-doped arrays. This may be due to the smaller tube diameter of $\mathrm{Cu}$-doped nanotubes and the higher crystallinity of $\mathrm{Cu}$-doped nanotube arrays. With the relatively large tube diameter, $\mathrm{Zn}$ doped nanotubes showed the most hydrophobic behaviour with the largest contact angle. As mentioned earlier, $\mathrm{Zn}$ may exist as $\mathrm{ZnO}$ in the structure of $\mathrm{Zn}$-doped $\mathrm{TiO}_{2}$. Previous studies pointed out that $\mathrm{ZnO}$ shows super hydrophobicity which may cause a significant reduction in the wettability of $\mathrm{TiO}_{2}$ nanotube arrays [33].

\subsection{Investigation of Biocompatibility of the Fabricated Nanotube Arrays}

For evaluation of the biocompatibility of anodized nanotube arrays, Vero cells were cultured on the surfaces of the pure and doped samples after annealing. Vero cells were grown on the surfaces of the samples at the initial density of 10,000 cells/sample. Cell proliferation as estimated by counting of cell numbers after 2-, 4-, and 7-days, was were found to be highest on Cu-doped nanotube arrays (Figure 6a) after control. Control refers to cell culture in its standard culturing conditions on a Petri dish. The superior performance of $\mathrm{Cu}$-doped nanotubes in terms of the regeneration of Vero cells could be attributed to their relatively small tube diameter and higher hydrophilicity. It has been previously reported that the nanotubes with a smaller diameter are more biocompatible than those with larger diameters [34]. In addition, it has been previously demonstrated that the presence of $\mathrm{Cu}$ ions in the lattice of $\mathrm{TiO}_{2}$ nanotubes enhances cell regeneration [25]. Cell counting showed that cell proliferation on $\mathrm{Cu}$-doped $\mathrm{TiO}_{2}$ arrays $(0.01 \mathrm{M})$ is higher than that of $\mathrm{Cu}$-doped $\mathrm{TiO}_{2}$ arrays $(0.01 \mathrm{M})$ as the former has smaller tube diameter and more contributions from $\mathrm{Cu}^{2+}$ ions.

Sr-doped and $\mathrm{Zn}$-doped $\mathrm{TiO}_{2}$ nanotube arrays have relatively similar tube diameters, while $\mathrm{Sr}$-doped $\mathrm{TiO}_{2}$ nanotubes show more support to cell regeneration indicating that the presence of strontium in the tubes has a greater effect on the cell growth. Although many previous studies pointed out that strontium doping enhances cell growth on $\mathrm{TiO}_{2}$ nanotube arrays, more cell growth on pure $\mathrm{TiO}_{2}$ nanotube was noticed 
in this study pointing to the dominating effect of tube diameter, as pure nanotubes have smaller diameter than those of Sr-doped nanotubes.

In order to observe the effect of doping on $\mathrm{TiO}_{2}$ nanotube arrays for the application of neural interfacing, neurons were cultured on the pure and doped nanotube arrays after annealing to evaluate the biocompatibility of doped nanotubes with the cultured neurons. Figure $6 \mathrm{~b}$ shows neurons number counted on fabricated nanotube arrays. Neurons with 25,000 initial number were cultured for 3-days. Doped nanotube arrays showed similar effects on the neurons as what was observed on the Vero cells. A previous study demonstrated that neurons show better growth on more conductive $\mathrm{TiO}_{2}$ nanotubes [35]. The results of our research are in line with this data. The largest number of neurons were found on $\mathrm{Cu}$-doped $\mathrm{TiO}_{2}(0.02 \mathrm{M})$ nanotubes which have the highest electrical conductivity among the fabricated arrays in this work. On the other hand, $\mathrm{Zn}$-doped $\mathrm{TiO}_{2}$ nanotubes which have the lowest electrical conductivity showed less support to cell growth than other doped nanotubes. To support these findings, the growth of neurons on the sample surfaces was inspected by SEM (Figure 7a-e). ImageJ software was used to estimate the surface areas of the samples which were covered by the neurons. The measurements showed that neurons cover about $46 \%$, $43 \%, 35 \%, 30 \%$ and $22 \%$ of the sample surfaces of $\mathrm{Cu}$-doped $\mathrm{TiO}_{2}(0.02 \mathrm{M})$, Cu-doped $\mathrm{TiO}_{2}(0.01 \mathrm{M})$, pure $\mathrm{TiO}_{2}$, Sr-doped $\mathrm{TiO}_{2}$ and $\mathrm{Zn}$-doped $\mathrm{TiO}_{2}$ nanotube arrays, respectively. The higher magnification SEM image shown in Figure $7 \mathrm{f}$ confirms direct attachment of a single neuron to the nanotubes.

\section{Conclusion}

In this paper, we report investigated the effect of doping on structure, electrochemical properties and biocompatibility of titania nanotube arrays for neural interface applications. Vertically oriented $\mathrm{TiO}_{2}$ nanotube arrays were doped with zinc, strontium and copper, separately during anodization. It was found that the doping changes the length, diameter and wall thickness of the nanotubes, which results in the observed differences in their electrochemical and biocompatibility properties. Besides, regeneration of Vero cells and neurons were found highest in $\mathrm{Cu}$-doped $\mathrm{TiO}_{2}$ nanotubes with relatively small tube diameter and higher hydrophilicity among the three types of dopants. Such improvements in the properties of $\mathrm{TiO}_{2}$ nanotube arrays make them a promising candidate electrode material architecture for the next generation of neural interfaces.

Acknowledgments: The content of this manuscript has been published in part as part of the thesis of Dhurgham Ismael Khudhair, "Nano electrode based on $\mathrm{TiO}_{2}$ nanotubes for neural interfacing, Deakin University, Institute for Intelligent Systems Research and Innovation, Victoria, Australia 2018."

\section{References}

[1] G. N. Angotzi, F. Boi, A. Lecomte, E. Miele, M. Malerba, S. Zucca, A. Casile, L. Berdondini, Sinaps: An implantable active pixel sensor CMOS-probe for simultaneous large-scale neural recordings, Biosensors and Bioelectronics 126 (2019) 355-364.

[2] S. Carli, L. Lambertini, E. Zucchini, F. Ciarpella, A. Scarpellini, M. Prato, E. Castagnola, L. Fadiga, D. Ricci, Single walled carbon nanohorns composite for neural sensing and stimulation, Sensors and Actuators B: Chemical 271 (2018) 280-288. 
[3] N. A. Kotov, J. O. Winter, I. P. Clements, E. Jan, B. P. Timko, S. Campidelli, S. Pathak, A. Mazzatenta, C. M. Lieber, M. Prato, et al., Nanomaterials for neural interfaces, Advanced Materials 21 (40) (2009) 3970-4004.

[4] D. Khudhair, S. Nahavandi, H. Garmestani, A. Bhatti, Microelectrode arrays: Architecture, challenges and engineering solutions, in: Emerging Trends in Neuro Engineering and Neural Computation, Springer (2017) 41-59.

[5] C. Xie, Z. Lin, L. Hanson, Y. Cui, B. Cui, Intracellular recording of action potentials by nanopillar electroporation, Nature nanotechnology 7 (3) (2012) 185-190.

[6] D. Br"uggemann, B. Wolfrum, V. Maybeck, Y. Mourzina, M. Jansen, A. Offenh"ausser, Nanostructured gold microelectrodes for extracellular recording from electrogenic cells, Nanotechnology 22 (26) (2011) 265104.

[7] D. B. Suyatin, L. Wallman, J. Thelin, C. N. Prinz, H. Jörntell, L. Samuelson, L. Montelius, J. Schouenborg, Nanowire-based electrode for acute in vivo neural recordings in the brain, PLoS one 8 (2) (2013) e56673.

[8] D. N. Heo, H.-J. Kim, Y. J. Lee, M. Heo, S. J. Lee, D. Lee, S. H. Do, S. H. Lee, I. K. Kwon, Flexible and highly biocompatible nanofiber-based electrodes for neural surface interfacing, ACS nano 11 (3) (2017) 2961-2971.

[9] Z. Yu, T. E. McKnight, M. N. Ericson, A. V. Melechko, M. L. Simpson, B. Morrison, Vertically aligned carbon nanofiber neural chip for interfacing with neurological system, $4^{\text {th }}$ IEEE International Conference on Nano/Molecular Medicine and Engineering (NANOMED) (2010) 188-191.

[10] Z. Lin, C. Xie, Y. Osakada, Y. Cui, B. Cui, Iridium oxide nanotube electrodes for sensitive and prolonged intracellular measurement of action potentials, Nature Communications 5 (2014) 3206

[11] C. I. Richards, K. Luong, R. Srinivasan, S. W. Turner, D. A. Dougherty, J. Korlach, H. A. Lester, Live-cell imaging of single receptor composition using zero-mode waveguide nanostructures, Nano letters 12 (7) (2012) 3690-3694.

[12] K. Wang, H. A. Fishman, H. Dai, J. S. Harris, Neural stimulation with a carbon nanotube microelectrode array, Nano letters 6 (9) (2006) 2043-2048.

[13] D. Khudhair, H. A. Hamedani, J. Gaburro, S. Shafei, S. Nahavandi, H. Garmestani, A. Bhatti, Enhancement of electro-chemical properties of $\mathrm{TiO}_{2}$ nanotubes for biological interfacing, Materials Science and Engineering: C 77 (2017) 111-120. 
[14] D. Khudhair, A. Bhatti, Y. Li, H. Hamedani, H. Garmestani, P. Hodgson, S. Nahavandi, Anodization parameters influencing the morphology and electrical properties of $\mathrm{TiO}_{2}$ nanotubes for living cell interfacing and investigations, Materials Science and Engineering: C 59 (2016) 1125-1142.

[15] C. A. Grimes, G. K. Mor, $\mathrm{TiO}_{2}$ nanotube arrays: synthesis, properties, and applications, Springer Science \& Business Media, 2009.

[16] M. Zhou, A. M. Glushenkov, O. Kartachova, Y. Li, Y. Chen, Titanium dioxide nanotube films for electrochemical supercapacitors: Biocompatibility and operation in an electrolyte based on a physiological fluid, Journal of The Electrochemical Society 162 (5) (2015) A5065-A5069.

[17] D. Khudhair, J. Gaburro, S. Shafei, A. Barlow, S. Nahavandi, A. Bhatti, Enhancement of electrochemical properties of micro/nano electrodes based on $\mathrm{TiO}_{2}$ nanotube arrays, Journal of Physics: Conference Series-Applied Nanotechnology and Neuroscience 829 (2017) 012010.

[18] M. Szkoda, A. Lisowska-Oleksiak, K. Siuzdak, Optimization of boron-doping process of titania nanotubes via electrochemical method toward enhanced photoactivity, Journal of Solid State Electrochemistry 20 (6) (2016) 1765-1774.

[19] D. Shikandar, N. Shetti, R. Kulkarni, S. Kulkarni, Silver-doped titania modified carbon electrode for electrochemical studies of Furantril , ECS Journal of Solid State Science and Technology 7 (7) (2018) Q3215-Q3220.

[20] N. P. Shetti, S. D. Bukkitgar, R. R. Kakarla, C. Reddy, T. M. Aminabhavi, ZnO-based nanostructured electrodes for electrochemical sensors and biosensors in biomedical applications, Biosensors and Bioelectronics (2019) 111-417.

[21] S. D. Bukkitgar, N. P. Shetti, R. M. Kulkarni, K. R. Reddy, S. S. Shukla, V. S. Saji, T. M. Aminabhavi, Electro-catalytic behavior of $\mathrm{Mg}$-doped $\mathrm{ZnO}$ nano-flakes for oxidation of antiinflammatory drug, Journal of The Electrochemical Society 166 (9) (2019) B3072-B3078.

[22] Y. Wang, D. Zhang, C. Wen, Y. Li, Processing and characterization of $\mathrm{SrTiO}_{3}-\mathrm{TiO}_{2}$ nanoparticlenanotube heterostructures on titanium for biomedical applications, ACS applied materials \& interfaces 7 (29) (2015) 16018-16026.

[23] H. A. Hamedani, N. K. Allam, H. Garmestani, M. A. El-Sayed, Electrochemical fabrication of strontium-doped $\mathrm{TiO}_{2}$ nanotube array electrodes and investigation of their photoelectrochemical properties, The Journal of Physical Chemistry C 115 (27) (2011) 13480-13486.

[24] S. Wang, K. Meng, L. Zhao, Q. Jiang, J. Lian, Superhydrophilic Cu-doped $\mathrm{TiO}_{2}$ thin film for solardriven photocatalysis, Ceramics International 40 (4) (2014) 5107-5110. 
[25] E. Wers, L. Bunetel, H. Oudadesse, B. Lefeuvre, A. Lucas-Girot, A. Mostafa, P. Pellen, Effect of copper and zinc on the bioactivity and cells viability of bioactive glasses, Bioceramics Development and Applications, (2013) doi:10.4172/2090-5025.S1-013

[26] B. Ghane-Motlagh, M. Sawan, A review of microelectrode array technologies: design and implementation challenges, $2^{\text {nd }}$ IEEE International Conference on Advances in Biomedical Engineering (ICABME) 2013 38-41.

[27] C. Kirkpatrick, Biological testing of materials and medical devices-a critical view of current and proposed methodologies for biocompatibility testing: cytotoxicity in vitro, Regulatory Affairs 4 (1) (1992) 13-32.

[28] M. Momeni, Y. Ghayeb, Z. Ghonchegi, Fabrication and characterization of copper doped $\mathrm{TiO}_{2}$ nanotube arrays by in situ electrochemical method as efficient visible-light photocatalyst, Ceramics International 41 (7) (2015) 8735-8741.

[29] M. Suhaimy, S. Abd Hamid, C. Lai, M. Hasan, M. Johan, $\mathrm{TiO}_{2}$ nanotubes supported $\mathrm{Cu}$ nanoparticles for improving photocatalytic degradation of simazine under UV illumination, Catalysts 6 (11) (2016) 167.

[30] H. Hamedani, N. Allam, M. El-Sayed, M. Khaleel, H. Garmestani, F. Alamgir, An experimental insight into the structural and electronic characteristics of strontium-doped titanium dioxide nanotube arrays, Advanced Functional Materials 24 (43) (2014) 6783-6796.

[31] P. Xiao, Y. Zhang, B. Garcia, S. Sepehri, D. Liu, G. Cao, Nanostructured electrode with titania nanotube arrays: fabrication, electrochemical properties, and applications for biosensing, Journal of nanoscience and nanotechnology 9 (4) (2009) 2426-2436.

[32] L. Sun, S. Zhang, X. Sun, X. He, Effect of the geometry of the anodized titania nanotube array on the performance of dye-sensitized solar cells, Journal of nanoscience and nanotechnology 10 (7) (2010) $4551-4561$.

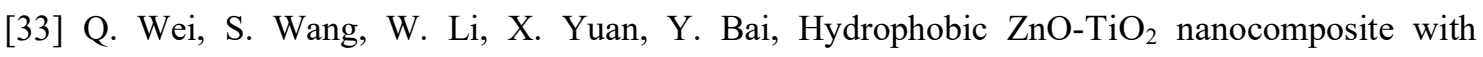
photocatalytic promoting self-cleaning surface, International Journal of Photoenergy (2015) 1-6.

[34] K. S. Brammer, S. Oh, C. J. Frandsen, S. Jin, Biomaterials and biotechnology schemes utilizing $\mathrm{TiO}_{2}$ nanotube arrays, Biomaterials Science and Engineering (2011) 193-210.

[35] J. A. Sorkin, Titania nanotube arrays as potential interfaces for neurological prostheses, Ph.D. thesis, Colorado State University (2014). 


\section{List of Figures:}

Figure 1: Top view SEM images of annealed nanotube arrays (a) pure $\mathrm{TiO}_{2}$, (b) $\mathrm{Zn}$-doped $\mathrm{TiO}_{2}$, (c) Srdoped $\mathrm{TiO}_{2}$, (d) Cu-doped $\mathrm{TiO}_{2}(0.01 \mathrm{M})$, (e) Cu-doped $\mathrm{TiO}_{2}(0.02 \mathrm{M})$ and (f) $\mathrm{Cu}$-doped $\mathrm{TiO}_{2}(0.03 \mathrm{M})$. The insets show cross-sectional SEM images of the samples.

Figure 2: XRD patterns of pure and doped $\mathrm{TiO}_{2}$ nanotube arrays after annealing.

Figure 3: High-resolution XPS spectra of pure and doped $\mathrm{TiO}_{2}$ nanotube arrays after annealing (a) $\mathrm{Ti}$ $2 \mathrm{p}$ in pure and doped $\mathrm{TiO}_{2}$ nanotube samples, (b) $\mathrm{Zn} 2 \mathrm{p}$ spectrum of $\mathrm{Zn}$-doped $\mathrm{TiO}_{2}$, (c) Sr 3d spectrum of $\mathrm{Sr}$-doped $\mathrm{TiO}_{2}$ and (d) $\mathrm{Cu} 2 \mathrm{p}$ spectrum of $\mathrm{Cu}$-doped $\mathrm{TiO}_{2}$ nanotube arrays.

Figure 4: Electrochemical properties of pure and doped $\mathrm{TiO}_{2}$ nanotube arrays after annealing (a) Bode impedance plots and (b) cyclic voltammograms obtained at scan rate of $100 \mathrm{mV} / \mathrm{sec}$.

Figure 5: Contact angle measurements showing degree of hydrophilicity of fabricated nanotube arrays (Error bars show standard deviation for $\mathrm{n}=5$ samples). The images of the water droplets on the fabricated nanotube arrays are shown above the chart.

Figure 6: (a) Vero cells proliferation results after 2-, 4-, and 7-days and (b) neuron cells number on the fabricated nanotube arrays after 3 -days (Error bars show standard deviation for $\mathrm{n}=3$ samples).

Figure 7: SEM images of neurons on the surface of (a) Cu-doped $\mathrm{TiO}_{2}(0.02 \mathrm{M})$, (b) Cu-doped $\mathrm{TiO}_{2}$ $(0.01 \mathrm{M})$, (c) pure $\mathrm{TiO}_{2}$, (d) Sr-doped $\mathrm{TiO}_{2}$, (e) $\mathrm{Zn}$-doped $\mathrm{TiO}_{2}$, and (f) higher magnification SEM image of a single neuron attached to $\mathrm{Cu}$-doped $\mathrm{TiO}_{2}(0.02 \mathrm{M})$ nanotube arrays. 
Figure 1

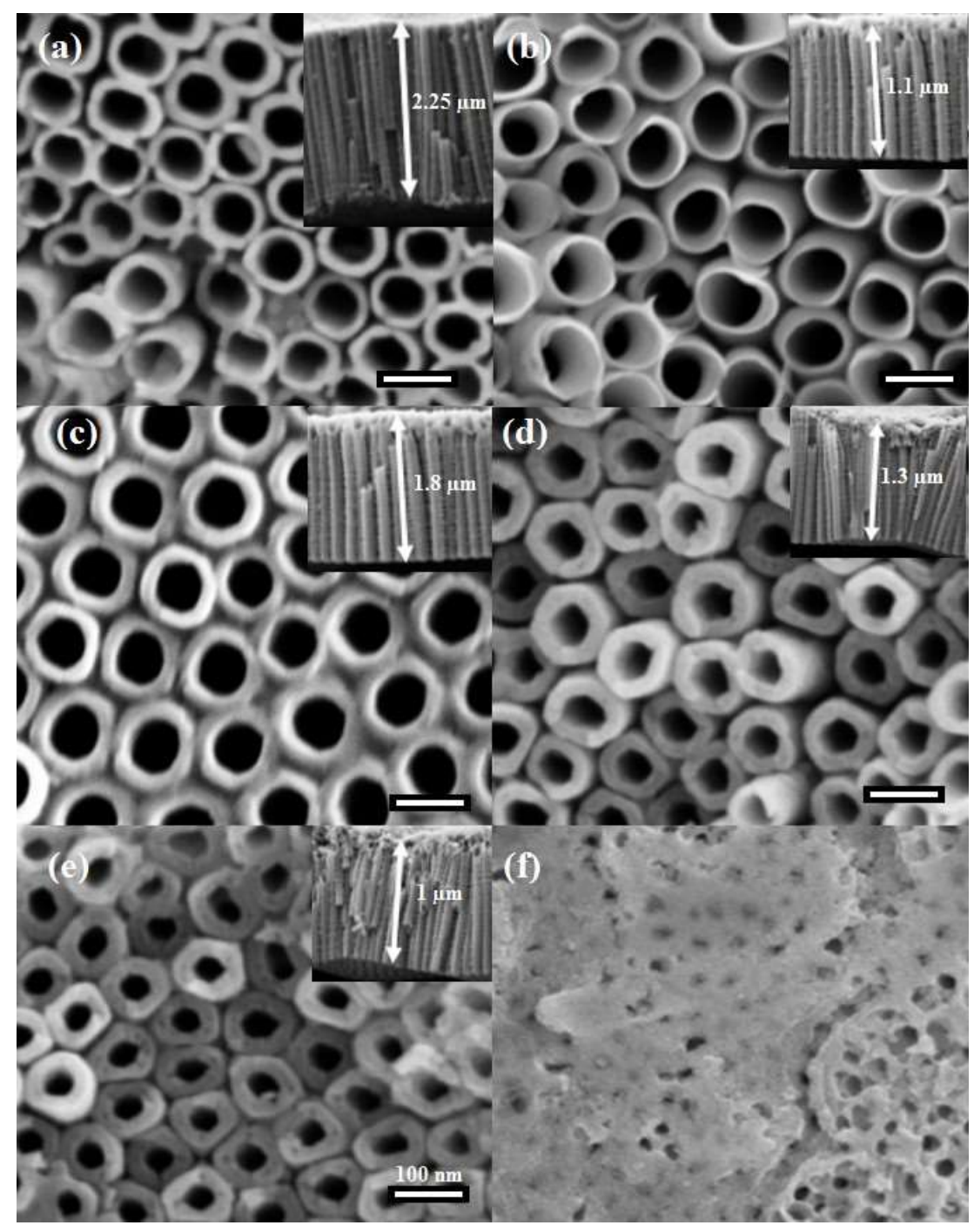


Figure 2

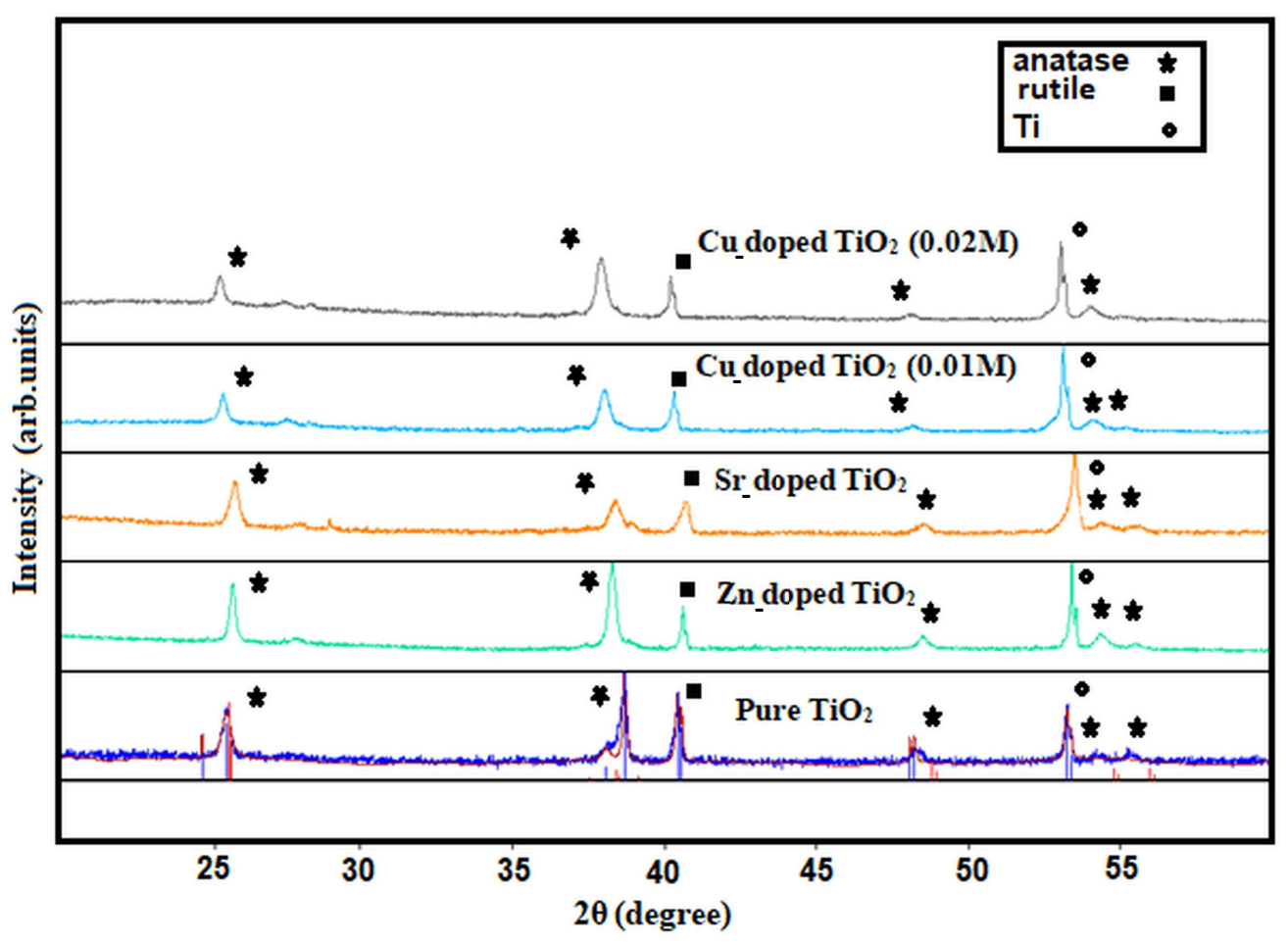


Figure 3
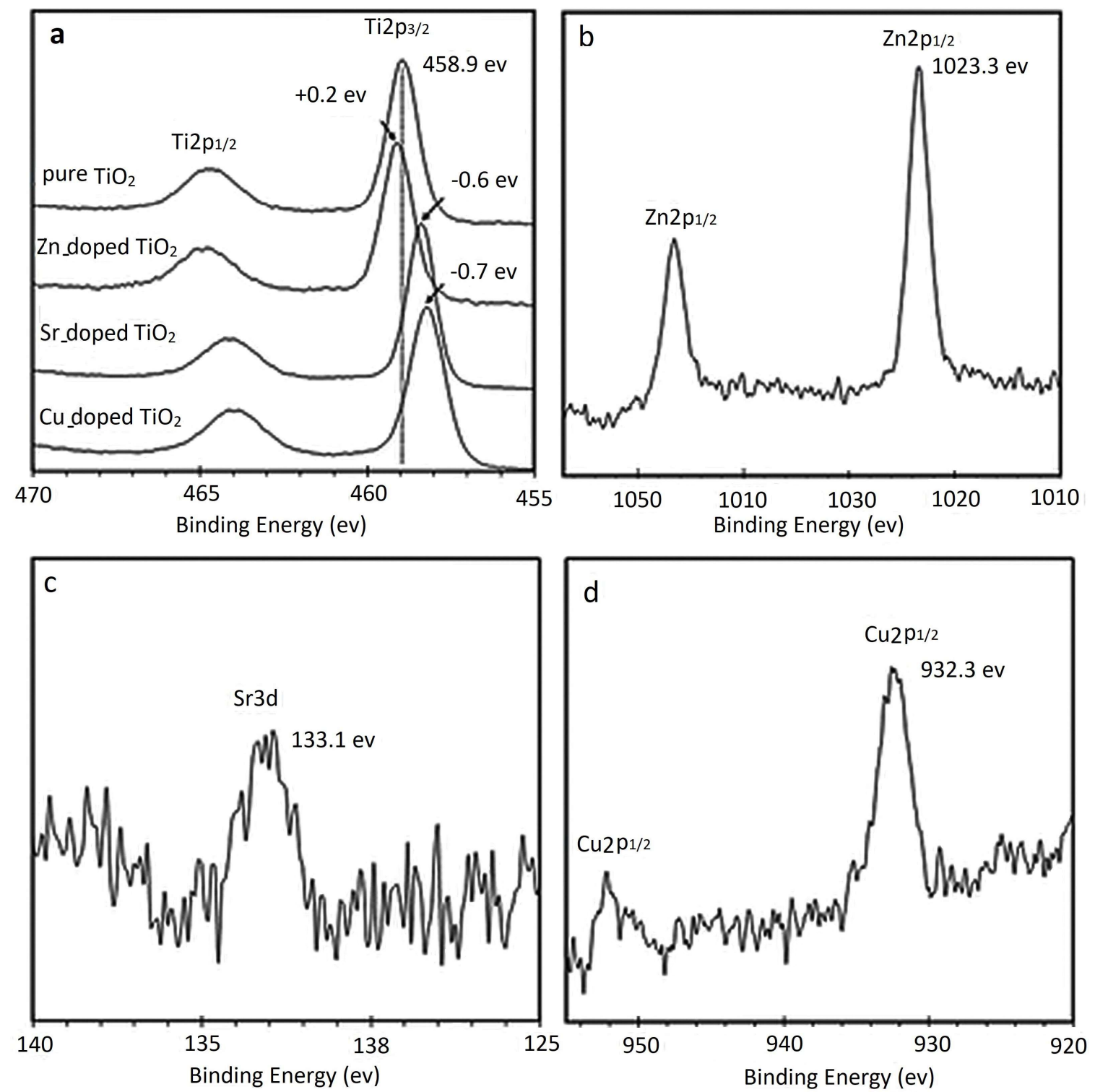
Figure 4

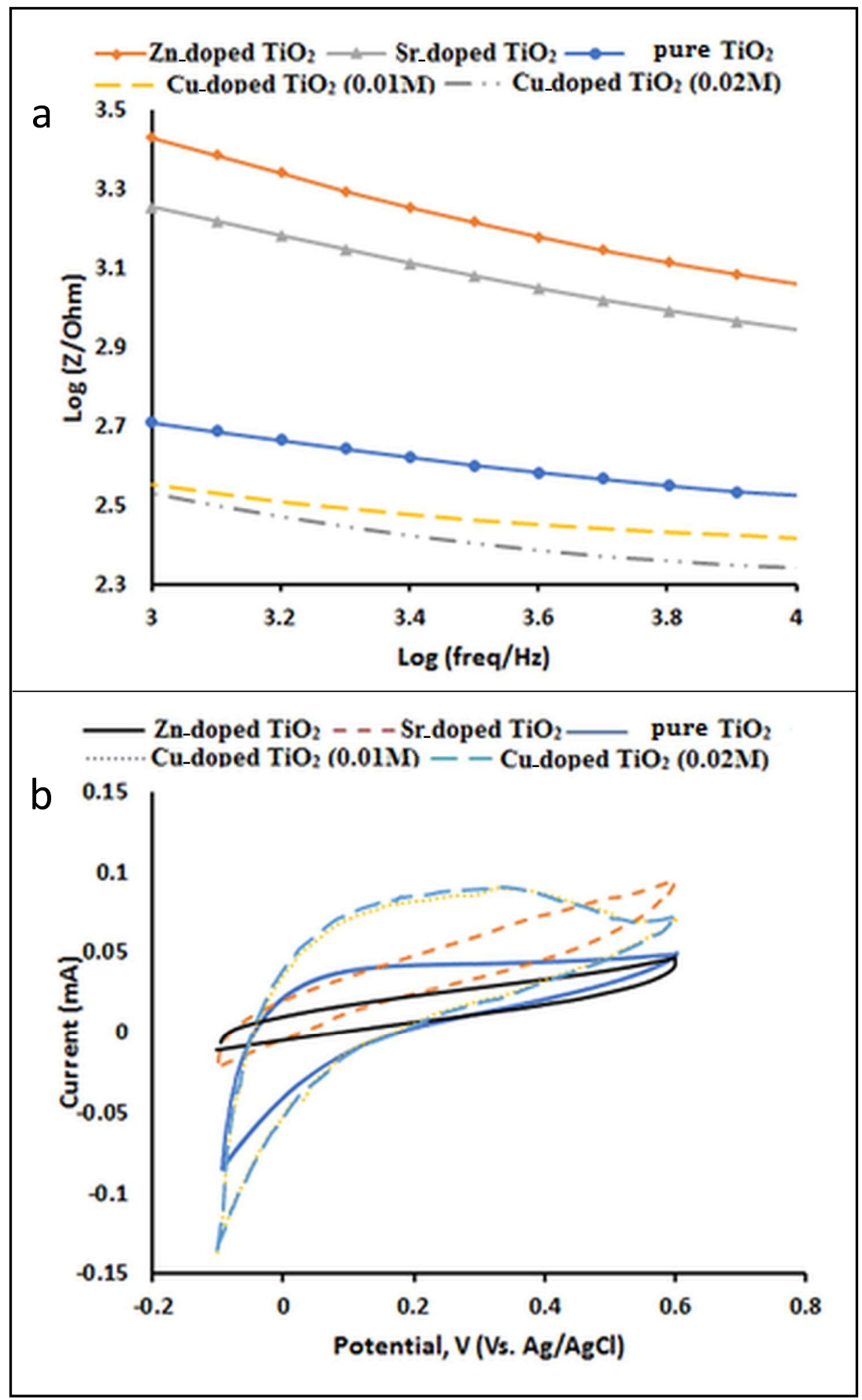


Figure 5

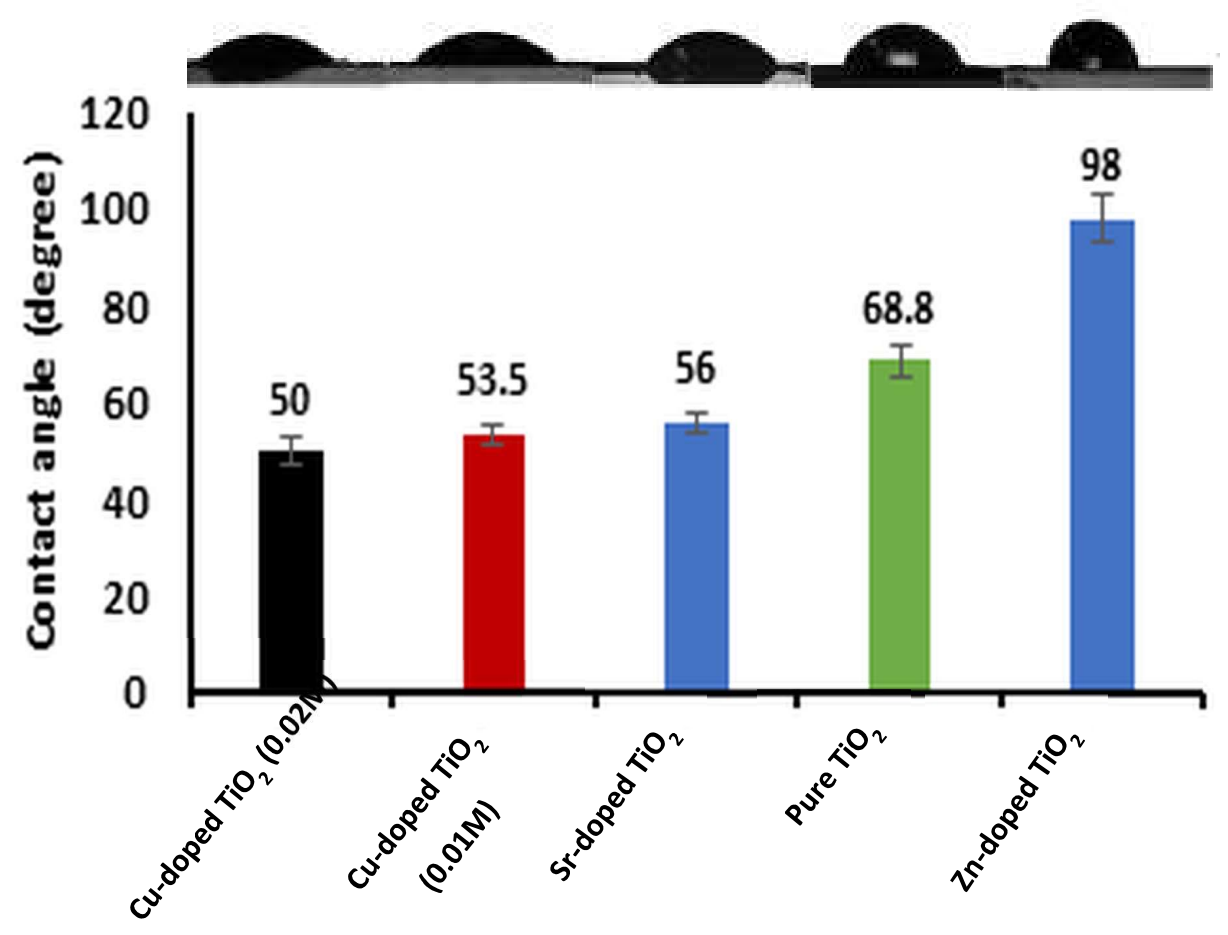


Figure 6

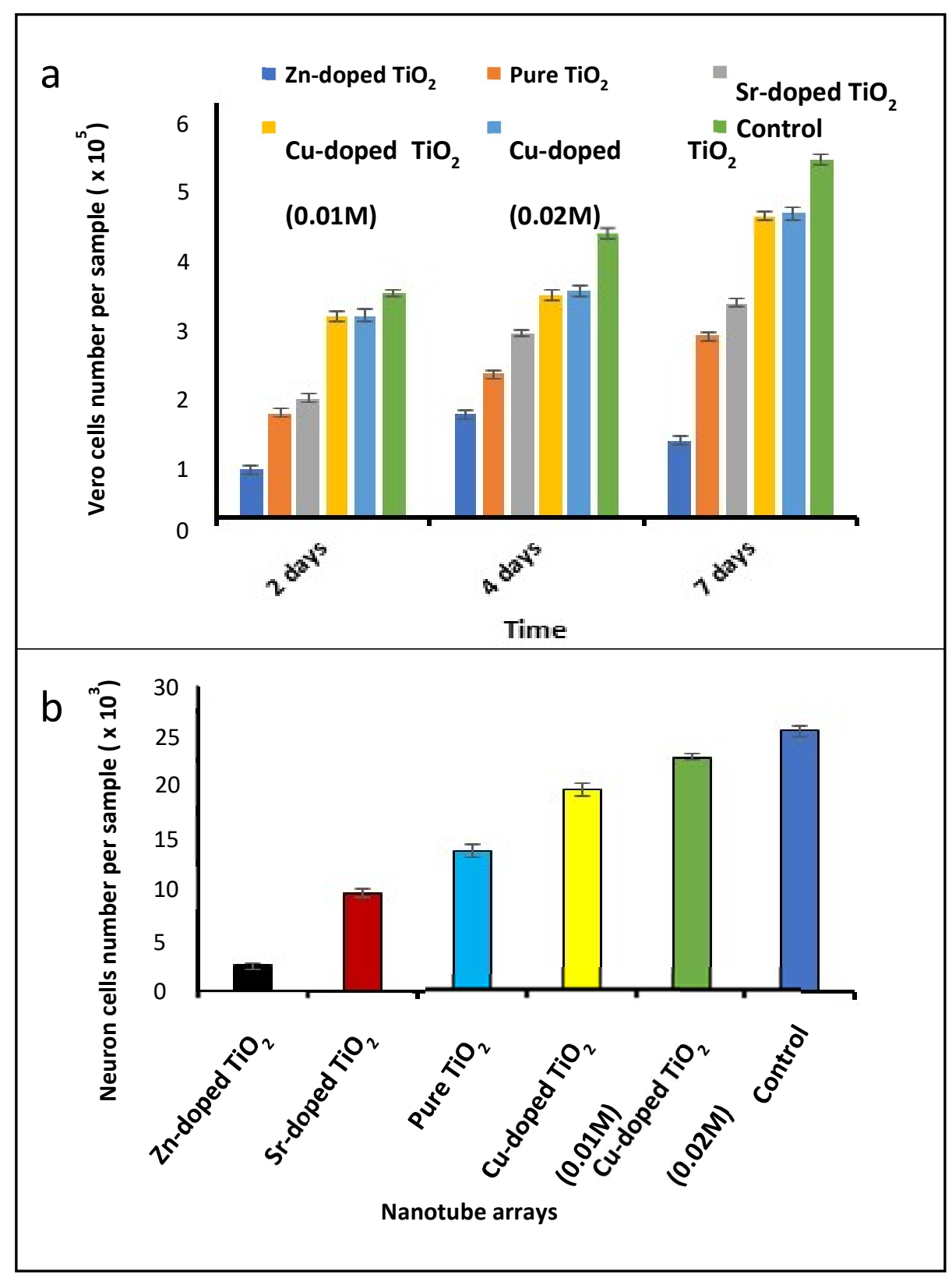


Figure 7

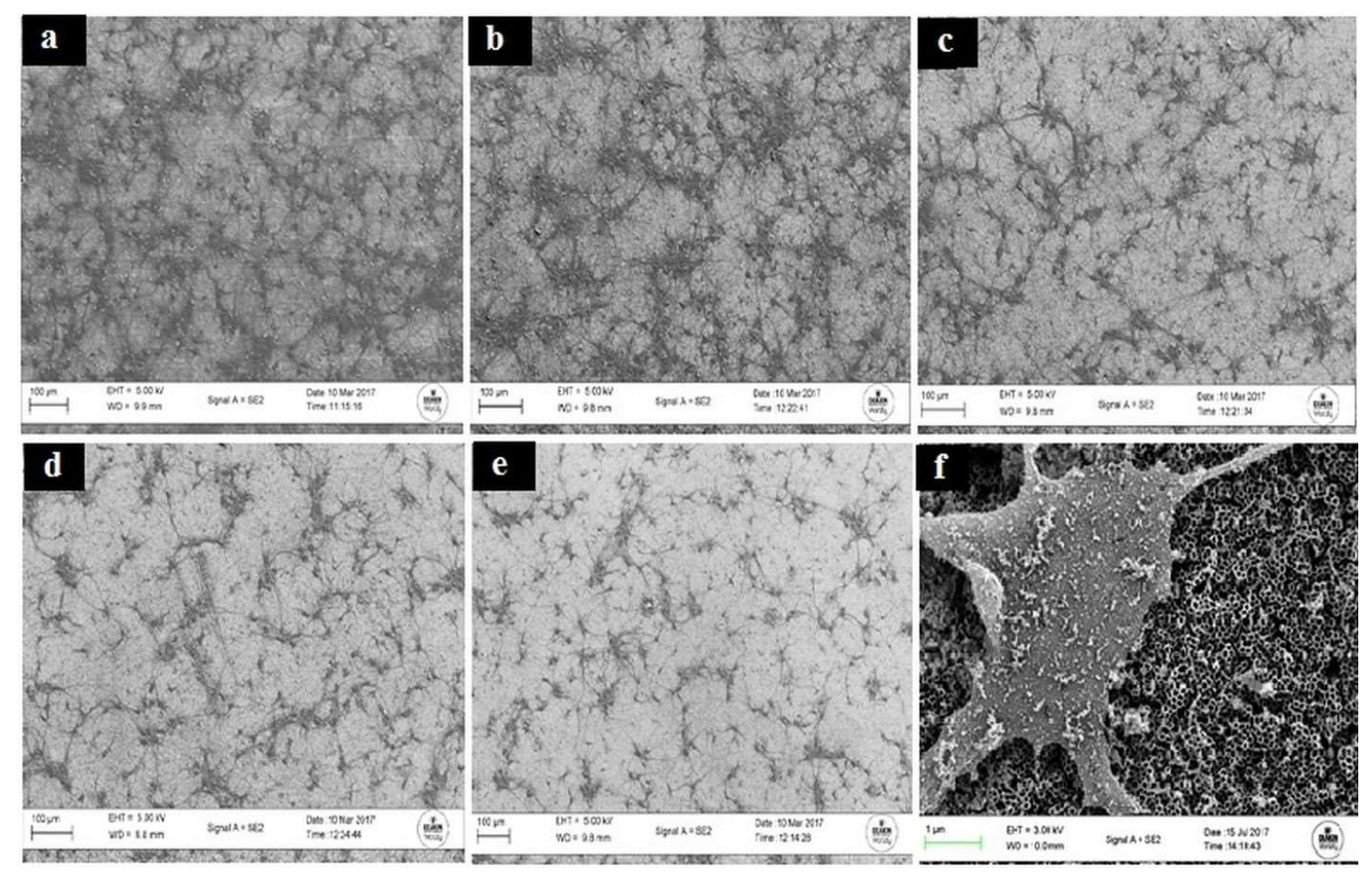

